\title{
Analise comparativa entre índices de vegetação e sua relação com o balanço hídrico em soja
}

\author{
Laura Pigatto Schaparini ${ }^{1}$, Denise Cybis Fontana ${ }^{1,2(*)}$, Genei Antonio Dalmago ${ }^{3}$, Grazieli Rodigheri ${ }^{2}$, \\ José Mauricio Cunha Fernandes ${ }^{3}$, Anderson Santi ${ }^{3}$ e Paulo Roberto Vargas ${ }^{4}$ \\ ${ }^{1}$ Universidade Federal do Rio Grande do Sul - Faculdade de Agronomia. Avenida Bento Gonçalves 7712, Caixa Postal 15096, CEP 91501.970. Porto Alegre, \\ RS.E-mails: laura_pigatto@yahoo.com.bre dfontana@ufrgs.br \\ ${ }^{2}$ Universidade Federal do Rio Grande do Sul, Programa de Pós-Graduação em Sensoriamento Remoto - PPGSR, Centro Estadual de Pesquisa em Senso- \\ riamento Remoto e Meteorologia - CEPSRM. Av. Bento Gonçalves, 9500 - Campus do Vale- Caixa Postal15044, CEP 91501-970 Porto Alegre, RS. E-mail: \\ grazielirodigheri@gmail.com \\ ${ }^{3}$ Embrapa Trigo. Rodovia BR 285, km 294, Caixa Postal 3081, CEP 99050-970 Passo Fundo, RS. E-mails: genei.dalmago@embrapa.br, mauricio.fernandes@ \\ embrapa.br e anderson.santi@embrapa.br \\ ${ }^{4}$ Fazenda Capão Grande, Carazinho, RS. E-mail: paulovargas@annex.com.br \\ ${ }^{(*)}$ Autor para correspondência.
}

\section{INFORMAÇÕES}

\section{História do artigo:}

Recebido em 17 de setembro de 2019

Aceito em 4 de março de 2020

Termos para indexação:

soja

condição hídrica

NDVI

EVI

evapotranspiração

\section{RESUMO}

A soja é uma leguminosa de ciclo anual e de grande importância econômica no Rio Grande do Sul. Para essa cultura, estresses provocados por deficiência hídrica são responsáveis pela instabilidade no rendimento e na produção de grãos. Uma das formas de monitorar a deficiência hídrica é por meio do sensoriamento remoto, o qual vem sendo amplamente utilizado na agricultura, muito frequentemente como índices de vegetação (IVs). O objetivo deste trabalho foi avaliar o padrão temporal dos índices NDVI e EVI para três safras de soja, com condições hídricas distintas, de forma a possibilitar a avaliação da relação entre IVs e as condições hídricas para a cultura. A área do experimento On Farm foi localizada no município de Carazinho RS. Na lavoura foi instalada uma estação meteorológica para medição de variáveis ambientais. Foi calculado o balanço hídrico diário para cada safra levando em consideração a evapotranspiração da cultura (ETc). Os IVs foram extraídos da plataforma Satveg. Além da análise gráfica, realizou-se análise de correlação entre os IVs e a ETc da soja. Tanto o NDVI quanto o EVI apresentaram forte relação com o desenvolvimento vegetativo das plantas, variando de forma semelhante ao longo do ciclo da soja. Ambos os índices tiverm o mesmo padrão de oscilações dos perfis nos períodos de deficiência hídrica. O NDVI e o EVI podem ser utilizados para avaliação da condição hídrica da cultura da soja.

(c) 2020 SBAgro. Todos os direitos reservados.

\section{Introdução}

A agricultura é uma das principais bases da economia do Brasil, abrangendo desde extensas monoculturas até a diversificação da produção em pequenas propriedades. 0 Rio Grande do Sul (RS) contribui, de forma marcante, para a definição da oferta nacional de alimentos. Cerca de $90 \%$ da área de cultivo é voltada à produção de grãos (cereais e 
oleaginosas), que se configura na principal atividade agrí) cola do Estado (Conab, 2018). A soja (Glycine max) é uma leguminosa de ciclo anual de primavera-verão que ocupa a maior área cultivada, sendo, portanto, a de maior importância econômica. Ao longo das últimas décadas, a produção de soja tem apresentado grande avanço, impulsionada não somente pelo aumento de área cultivada, mas também pela aplicação de técnicas de manejo avançadas, que tem permitido o incremento na produtividade (Freitas, 2011).

As condições meteorológicas quando adversas são aquelas que, com maior frequência, determinam limitações no incremento de biomassa e, consequentemente, da produtividade agrícola. Segundo Hoogenboom (2000), dentre todas as variáveis meteorológicas, a radiação solar, a temperatura do ar e a precipitação pluvial são as que mais determinam o crescimento, o desenvolvimento e a produtividade das culturas. Porém, no Rio Grande do Sul, a variabilidade na precipitação pluvial é a responsável pelos maiores impactos negativos às culturas produtoras de grãos. Para Bergamaschi et al (2003), a variação sazonal da condição hídrica no estado é causada principalmente pela maior demanda evaporativa da atmosfera no verão que, via de regra supera a precipitação pluvial. Nestas condições é comum a ocorrência de déficit hídrico, o qual se desenvolve quando a absorção de água do solo é menor do que a transpiração das plantas.

Para a soja, cujo os períodos críticos de desenvolvimento ocorrem eminentemente no período de verão, estresses provocados por deficiência hídrica são responsáveis pela instabilidade na produtividade e na produção de grãos do Estado, sendo muito variável de um ano para o outro (Berlato \& Fontana, 1999). Dependendo do nível de estresse hídrico e do estádio fisiológico em que as plantas se encontram, diferentes processos biológicos podem ser afetados, causando importante variabilidade no rendimento de grãos da cultura e na qualidade do grão (Arias et al., 2015), o que se constitui em um dos principais problemas de perdas do setor.

A evapotranspiração (ET) é o processo biofísico pelo qual a energia do ambiente é trocada entre a superfície e a atmosfera através da evaporação direta da água do solo, da evaporação da água disponível em outras partes do sistema e da água que passa pelo dossel de plantas via processo de transpiração. É, portanto, um componente crítico ao desenvolvimento de vários processos físicos e biológicos dentro de um sistema de produção agrícola. Por exemplo, no desenvolvimento das plantas a evapotranspiração pode afetar, direta e indiretamente, processos em escala celular, foliar, copa e dossel da cultura (Katul et al., 2012). Dada a estreita relação entre ET e o crescimento e desenvolvimento das plantas (Monteiro, 2009), esta variável tem sido muito utilizada na elaboração de modelos de estimativa de produtividade para várias culturas de impor- tância agrícola (Melo et al., 2004). Tais modelos, frequentemente, esbarram na necessidade de uma representação espacial mais detalhada dos rendimentos de grãos, do que aquela possível de ser obtida a partir de dados oriundos de estações meteorológicas de superfície, necessitando de outras estratégias de abordagem.

Uma das ferramentas que vem sendo amplamente utilizada na agricultura, com potencial de ampliar a amostragem de respostas de plantas, de forma mais detalhada geograficamente (Melo et al., 2008), é o sensoriamento remoto (SR). Os dados obtidos por SR podem servir de importante subsídio para a modelagem de crescimento e desenvolvimento das culturas, até mesmo da estimativa do rendimento de grãos das mesmas. Isso decorre de uma ampla gama de opções na hora da escolha do sensor, possibilitando a escolha de bandas espectrais específicas para a avaliação de processos específicos, ou momentos distintos do desenvolvimento de uma cultura (Jensen, 2011).

Nos estudos com SR é muito frequente o uso dos dę nominados índices de vegetação (IV). Os IVs são medidas radiométricas adimensionais que indicam a abundância relativa e a atividade da vegetação verde, incluindo índice de área foliar (IAF), porcentagem de cobertura verde, teor de clorofila, biomassa verde e radiação fotossinteticamente ativa. Entre as muitas possibilidades de equações disponíveis na literatura para este fim, o NDVI (do inglês Normalized Difference Vegetation Index) é o mais utilizado. Outro IV com potencial de uso para acompanhamento da dinâmica da vegetação é o Enhanced Vegetation Index (EVI), o qual foi proposto posteriormente ao NDVI, por Huete et al. (2002). O EVI é um NDVI modificado desenvolvido para promover a redução das influências atmosféricas e do solo de fundo do dossel no monitoramento da vegetação. As fases de crescimento e desenvolvimento das culturas anuais apresentam valores de IVs distintos ao longo do ciclo, caracterizando os denominados perfis temporais dos IVs (Risso et al., 2009). Estes podem ser úteis em sistemas de monitoramento para identificar o impacto de eventos adversos em estádios fenológicos críticos.

Sabendo-se da importância econômica da soja para o $\mathrm{RS}$, e do fato de que um dos principais fatores de variabilidade na produtividade é a deficiência hídrica e, ainda, que é possível monitorá-la usando índices de vegetação, o objetivo deste trabalho foi analisar o padrão temporal dos índices NDVI e EVI para três safras soja com condições hídricas distintas durante o ciclo, de forma a possibilitar a avaliação da relação entre IVs e as condições hídricas para a cultura.

\section{Material e Métodos}

0 experimento foi conduzido em formato On Farm, em uma área comercial de uma propriedade de 553,7 hectares, localizada no Município de Carazinho, RS sob as coorde- 
nadas $28^{\circ} 13^{\prime} 46^{\prime \prime} \mathrm{S}$ e $52^{\circ} 54^{\prime} 32^{\prime \prime}$, nas safras 2015-2016, 20162017 e 2017-2018. O clima da região segundo a classificação climática de Köppen, é Cfa ou seja, não há ocorrência de estação seca e as precipitações pluviais são distribuídas ao longo do ano (Alvares et al., 2013). O solo da área é um Latossolo Vermelho Aluminoférrico tipico (Streck et al., 2008), profundo, com boa drenagem natural, adequado às culturas produtoras de grãos.

A semeadura da soja ocorreu em 16/11/2015, 8/11/2016 e 13/11/2017, com emergência das plantas em 24/11/2015, 16/11/2016 e 21/11/2017 e colheita em 24/03/2016, 24/03/2017 e 03/04/2018, respectivamente, para as três safras. Os genótipos utilizados em cada safra foram: BMX Apolo RR, NS 5445 IPRO e DM 5958 RSF IPRO, nas safras de 2015, 2016 e 2017, respectivamente, todos com hábito de crescimento indeterminado e pertencentes aos grupos de maturidade relativa: 5.5, 5.4 e 5.8, respectivamente. 0 espaçamento adotado foi de $45 \mathrm{~cm}$ entre linhas em todas as safras. A população final de plantas foi de 26,9, 26,8 e 23,9 plantas $\mathrm{m}^{-2}$. A adubação de base foi realizada conforme análise de solo e indicação de alto rendimento de grãos para a cultura, com aplicação de $281 \mathrm{~kg} \mathrm{ha}^{-1}$ de NPK, da fórmula 05-20-20, em 2015, $304 \mathrm{~kg} \mathrm{ha}^{-1}$ de NPK, da fórmula 0428-08, mais $171 \mathrm{~kg} \mathrm{ha}^{-1}$ de $\mathrm{KCl}$, em 2016 e $300 \mathrm{~kg} \mathrm{ha}^{-1}$ de NPK da fórmula 04-28-08 mais $160 \mathrm{~kg} \mathrm{ha}^{-1}$ de $\mathrm{KCl}$, em 2017. Os controles fitossanitários foram realizados conforme indicações técnicas e necessidade para o cultivo da soja.

Os dados de NDVI (Equação 1) e EVI (Equação 2) foram extraídos através da plataforma SATveg (Sistema de Análise Temporal da Vegetação) (SATVeg, 2019) que é uma ferramenta Web desenvolvida pela Embrapa Informática Agropecuária, destinada ao acesso e visualização de perfis temporais dos índices vegetativos NDVI e EVI do sensor MODIS em qualquer local da América do Sul.

$$
\begin{aligned}
& N D V I=\frac{\rho I V-\rho V}{\rho I V+\rho V} \\
& E V I=2,5\left(\frac{\rho I V-\rho V}{\rho I V+\rho V-7,5 \rho A z u l+1}\right)
\end{aligned}
$$

Onde, $\rho$ IV a reflectância no comprimento de onda do infravermelho próximo e $\rho \mathrm{V}$ a reflectância do vermelho e $\rho$ Azul a reflectância no comprimento de onda do azul.

Os dados meteorológicos foram coletados através de estação meteorológica instalada na lavoura, com sensores posicionados a 1,5 $\mathrm{m}$ de altura do solo para as três safras de soja. Para a medição da temperatura e umidade relativa do ar foi utilizado um sensor conjugado, modelo HC2S3-L da Campbell Scientific, Inc. A radiação solar global incidente foi medida com um piranômetro modelo SP-110-L-10, marcar Apogee. $\mathrm{O}$ vento foi medido com um anemômetro sônico bidirecional, modelo WINDSONIC1-L34, marca GILL. A precipitação pluvial (P) foi medida com o pluviômetro TB4-L da Campbell Scientific, Inc. Todos os sensores foram conectados a um datalogger CR 1000, da Campbell Scientific, Inc. e as leituras foram feitas a cada 30 segundos, com médias e/ou totalização, armazenadas a cada 15 minutos, conforme a variável. o preenchimento de falhas quando ocorreram foi feita com dados meteorológicos registrados na estação meteorológica da Embrapa Trigo, pertencente ao $8^{\circ}$ Distrito de Meteorologia (8ํㅡㄹ DISME) do Instituto Nacional de Meteorologia (INMET).

A partir dos dados medidos foi calculada a evapotranspiração potencial (ETP) com o método de Penam-Montheith (1989). Para o cálculo da evapotranspiração da cultura (ETc), a ETP calculada foi multiplicada pelo coeficiente de cultura (Kc), cujos valores variaram de 0,56 a 1,50 (Farias et al, 2001), expressando as variações no consumo hídrico de cada fase fenológica da cultura. Com a ETc foi realizado o cálculo do balanço hídrico meteorológico diário, pelo método desenvolvido por Thornthwaite \& Mether (1955) demonstrado por Cunha et al. (1992), ao longo do ciclo da soja para cada safra. O cálculo foi composto pelas variáveis meteorológicas P, ETc e a partir destas, foi calculada a diferença entre P e ETc e o negativo acumulado, que representa a perda potencial de água quando $\mathrm{P}$ não atende à demanda evaporativa da cultura. Para a capacidade de armazenamento de água no solo (CAD) foi utilizado o valor de $150 \mathrm{~mm}$, para solos argilosos com culturas de raízes de moderada profundidade.

Os dados de IVs do sensor MODIS são gerados a partir de uma composição de imagens a cada 16 dias. Para realizar uma melhor comparação entre os dados meteorológicos e IVs, os valores de precipitação pluvial (mm) medidos e os valores de ETc ( $\mathrm{mm})$, excesso e deficiência hídrica (mm), oriundos do balanço hídrico diário foram somados para períodos de 16 dias. Sendo que, NOV_1 (01/11 a 16/11/2017), NOV_2 (17/11 a 02/12/2017), DEZ_1 (02/12 a 18/12/2017), DEZ_2 (19/12/2017 a 03/01/2018), JAN_1 (04/01 a 19/01/2018), JAN_2 (20/01 a 04/02/2018), FEV_1 (05/02 a 20/02/2018), FEV_2 (21/02 a 08/03/2018), MAR_1 (09/03 a 24/03/2018) e MAR_2 (25/03 a 09/04/2018). Os dados do balanço hídrico diário e dos IVs foram avaliados graficamente e analisados de acordo com a variação do padrão temporal ao longo da safra. Por fim, os dados de ETc, NDVI e EVI foram separados levando em consideração o período inicial do desenvolvimento vegetativo, de início de novembro até o final do mês de dezembro (subperíodo vegetativo) e pleno desenvolvimento, de início de dezembro até o final do ciclo (Subperíodo reprodutivo), para realização de análise de correlação de Pearson, com significância estatística de 95\% e 99\%. 


\section{Resultados e Discussão}

A Figura 1 mostra o padrão de variação do NDVI e EVI ao longo da safra da soja para os três anos avaliados. Verifica-se que a evolução temporal dos mesmos mostrou algumas similaridades, o que é típico de culturas anuais (Junges \& Fontana, 2009; Risso et al., 2012; Pinto et al., 2017). Em novembro, quando ocorreu a semeadura da soja, os valores dos IVs foram baixos e, posteriormente, mostraram crescimento até atingir o seu máximo nos meses de janeiro e fevereiro. Nestes meses, a soja encontrava-se no seu desenvolvimento vegetativo máximo, considerando as datas de semeadura de novembro. Em Final de março a início de abril os valores dos IVs decresceram rapidamente, representando o período de maturação e de colheita da soja (Couto, 2012). Apesar destas similaridades, foram observadas, em ambos índices, importantes diferenças entre as safras avaliadas, as quais possivelmente tenham sido decorrentes das condições hídricas distintas, uma vez que os materiais genéticos utilizados foram todos de crescimento indeterminado e grupo de maturação próximos.

Na Figura 2 estão representados graficamente os perfis temporais dos dois IVs e também o Balanço Hídrico diário para cada período analisado, de forma a dar subsídios às análises.

Para as safras 2015-2016 e 2016-2017, o balanço hídrico evidenciou a ocorrência de deficiências hídricas em alguns períodos ao longo do ciclo da cultura da soja. Na safra 2015-2016 a deficiência ocorreu durante o mês de janeiro, enquanto que na safra 2016-2017 foi no final do mês de dezembro e no final do mês de janeiro e início de fevereiro. Os IVs, NDVI e EVI, responderam a esses períodos de baixa disponibilidade hídrica com redução nos valores. Nestas duas safras o perfil dos IVs não apresentou o padrão conn tínuo de evolução temporal (Fiori et al., 2015) como freł quentemente se observa em culturas anuais com adequada
Figura 2. Componentes do balanço hídrico meteorológico durante as três safras analisadas. Excesso Hídrico, deficiência hídrica, evapotranspiração da cultura (ETc), precipitação pluvial. Índices de vegetação Normalized Difference Vegetation Index (NDVI) e Enhanced Vegetation Index (EVI) ao longo do crescimento e desenvolvimento das plantas de soja em três safras no período de 2015 a 2018 [NOV_1 (01/11 a 16/11/2017), NOV_2 (17/11 a 02/12/2017), DEZ_1 (02/12 a 18/12/2017), DEZ_2 (19/12/2017 a 03/01/2018), JAN_1 (04/01 a 19/01/2018), JAN_2 (20/01 a 04/02/2018), FEV_1 (05/02 a 20/02/2018), FEV_2 (21/02 a 08/03/2018), MAR_1 (09/03 a 24/03/2018) e MAR_2 (25/03 a 09/04/2018)]. Carazinho - RS.
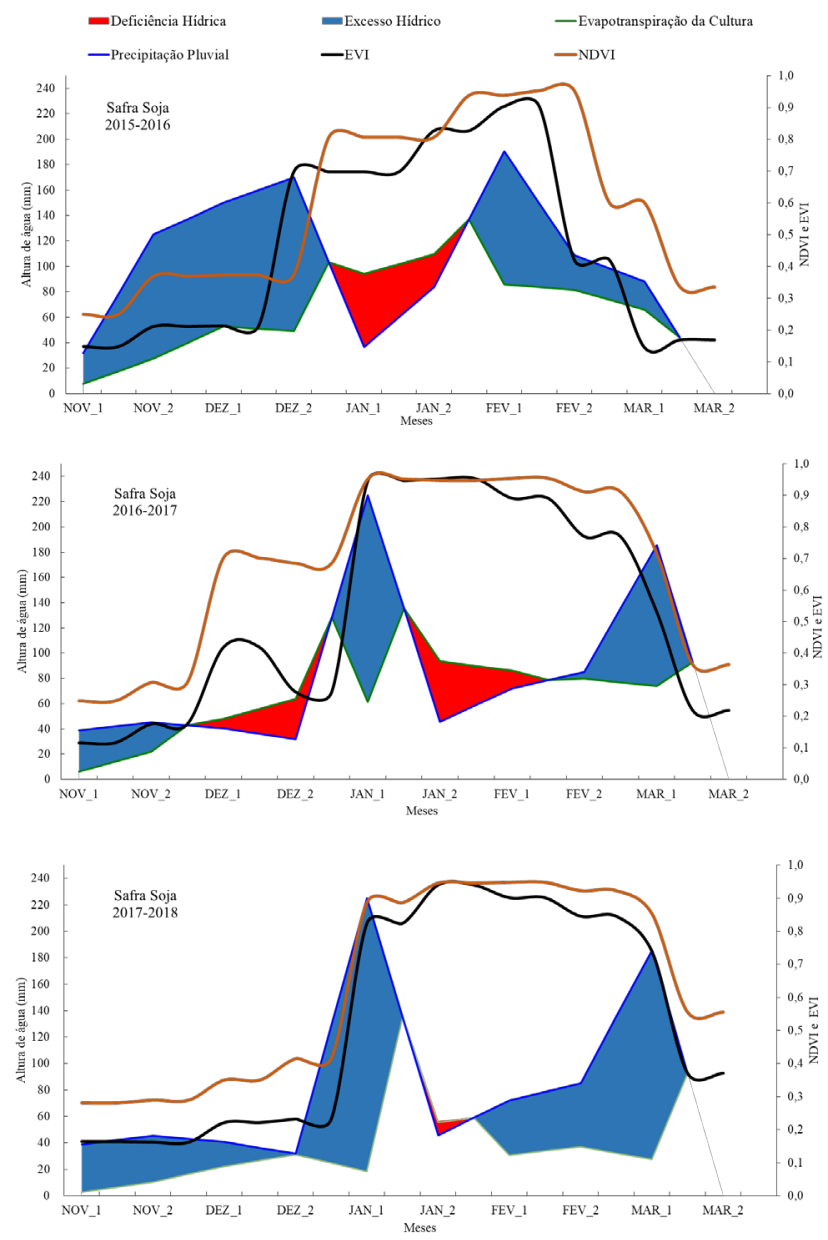

Figura 1. Perfil temporal dos índices de vegetação Normalized Difference Vegetation Index (NDVI) e Enhanced Vegetation Index (EVI) ao longo do crescimento e desenvolvimento das plantas de soja em três safras no período de 2015 a 2018 [NOV_1 (01/11 a 16/11/2017), NOV_2 (17/11 a 02/12/2017), DEZ_1 (02/12 a 18/12/2017), DEZ_2 (19/12/2017 a 03/01/2018), JAN_1 (04/01 a 19/01/2018), JAN_2 (20/01 a 04/02/2018), FEV_1 (05/02 a 20/02/2018), FEV_2 (21/02 a 08/03/2018), MAR_1 (09/03 a 24/03/2018) e MAR_2 (25/03 a 09/04/2018)]. Carazinho, RS.
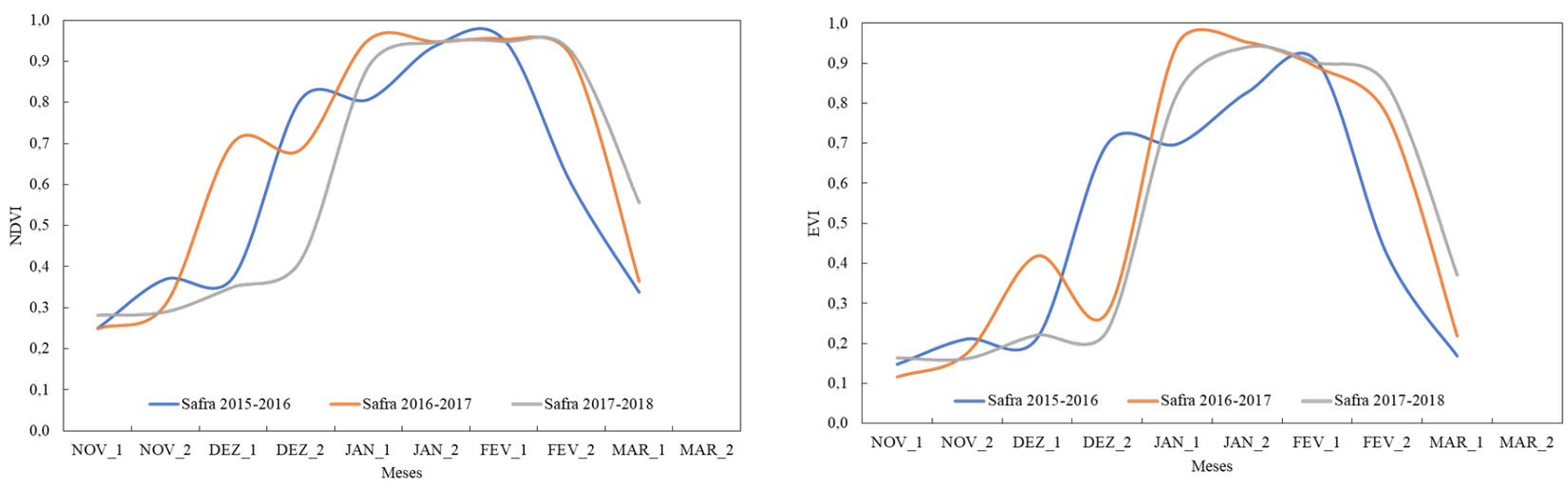
condição hídrica. Mas é importante ressaltar que, segundo Cunha (2017), períodos de deficiência hídrica no início do ciclo não acarretam necessariamente em perda de rendimentos de grão de soja na região de Passo Fundo.

Já na safra 2017/2018, o perfil de NDVI e EVI mostraram o padrão típico de variação de culturas anuais. Ao compararmos os perfis dos IVs com o balanço hídrico desta safra, observou-se evolução contínua e suave no tempo, o que pode ser explicado pelas condições hídricas do período, em que não ocorreram deficiências hídricas relevantes para as plantas (Figura 2). Segundo Roppa Kuss et al. (2008), lavouras de soja com disponibilidade hídrica adequada, seja por meio de irrigação ou precipitação pluvial ao longo de todo o ciclo, apresentam maiores rendimentos de grãos do que lavouras com disponibilidade de água irregular e abaixo da necessidade da cultura. Um período de estiagem pode estar relacionado a hidrologia do local (por exemplo, precipitação pluvial, umidade do solo), condição da planta (por exemplo, tipo, idade, estádio de crescimento e vigor, incluindo clorofila conteúdo e índice de área foliar que estão relacionados aos IVs) (Joiner et al., 2018).

Na Tabela 1 estão apresentados os valores de correlação entre ETc e os índices NDVI e EVI para as três safras analia sadas. Os valores foram divididos entre os subperíodos vegetativos e reprodutivos da soja. Na Figura 3 encontram-se a dispersão dos dados para estes mesmos subperíodos.

No subperíodo vegetativo (início de novembro a final de dezembro) a ETc mostrou correlações positivas e significativas com ambos IVs (Tabela 1). Uma correlação positiva explica que o aumento de valor de uma variável pode ser explicado pelo aumento do valor de outra variável, ou seja, quanto maiores os IVs maior a ETc. Como, segundo Jensen (2011), os IVs são indicadores de biomassa verde e é esperado que, em condições de maior biomassa verde, a ETc daquele dossel seja maior. Apesar das correlações significativas em ambos IVs, o NDVI apresentou correlação mais forte (99\%) e menor dispersão dos dados com a ETc, do que o EVI.

Já as correlações no subperíodo seguinte, quando as plantas se encontravam na fase reprodutiva, evidenciou-se que, especialmente, o NDVI tendeu a saturação, sendo esta uma das principais limitações deste índice (Jensen, 2011; Almeida, 2008). O termo saturação é usado para os casos em que aumentos ou diminuições na biomassa não resultam em consequentes variações no NDVI. Estudos vem demostrando que, para a cultura da soja, a saturação do NDVI ocorre em IAF acima de 5,0 (Fontana et al., 2019), o que é característico do subperíodo reprodutivo da cultura. As correlações realizadas nesse subperíodo do desenvolvimento da soja foram baixas e negativas, decorrentes do fato de que, enquanto a ETc continua apresentando mudanças em função das condições de demanda hídrica da atmosfera e de incrementos de IAF, os IVs se mostram estabilizados em seus valores máximos, saturados. Essa condição explica as baixas correlações entre ETc e IVs, quando se considera o ciclo todo da cultura, em que a correlação foi significativa apenas para o NDVI.

\section{Conclusão}

Os índices de vegetação, NDVI e EVI, podem ser utilizados como indicadores do desenvolvimento da soja ao longo da safra. Períodos de deficiência hídrica, demonstrados pelo balanço hídrico, acarretam em oscilações no perfil temporal dos IVs ao longo do ciclo da cultura. Safras de soja em que ocorrem pequenos ou nulos períodos de deficiência hídrica, tendem a apresentar perfis de NDVI e EVI mais contínuos. A ETc apresenta forte correlações com os IVs, reforçando que o desenvolvimento vegetativo adequado da soja, depende, principalmente, das condições hídricas ofertadas à lavoura.

\section{Agradecimento}

O presente trabalho foi realizado com apoio da Conselho Nacional de Desenvolvimento Científico e Tecnológico - Brasil (CNPq) - Código de Financiamento 001 e pela Embrapa Trigo, por meio do projeto SEG 02.15.07.003.00.00. A equipe agradece também aos funcionários da Embrapa Trigo, Elisson S. S. Pauletti e Cristian M. Plentz, que auxiliaram na condução do experimento e coletas dos dados e a Granja Campão Grande pela parceria no trabalho.

Tabela 1. Coeficiente de correlação de Pearson entre a Evapotranspiração da cultura (ETc) e os Índices de vegetação (IVs) Normalized Difference Vegetation Index (NDVI) e Enhanced Vegetation Index (EVI) de todas as safras em conjunto (2015-2016, 2016-2017, 2017-2018) para o subperíodo vegetativo, subperíodo reprodutivo e ciclo completo da cultura. Carazinho, RS, 2019. Correlações com significância estatística $(* p>0,05 ; * * p>0,001 ;$ ns = não significativo)

\begin{tabular}{cccc}
\multicolumn{2}{c}{ Evapotranspiração da Cultura } & \multicolumn{2}{c}{ Ciclo Completo } \\
IVs & Subperiodo vegetativo & Subperíodo reprodutivo & $0,35^{*}$ \\
NDVI & $0,86^{* *}$ & $-0,19 \mathrm{~ns}$ & $0,23 \mathrm{~ns}$
\end{tabular}


Figura 3. Relação entre a Evapotranspiração da cultura e os Índices de vegetação Normalized Difference Vegetation Index (NDVI) e Enhanced Vegetation Index (EVI) de todas as safras em conjunto (2015-2016, 2016-2017, 2017-2018) para o subperíodo vegetativo $(a, b)$, subperíodo reprodutivo (c, d) e ciclo completo da cultura (e, f). Carazinho, RS, 2019.
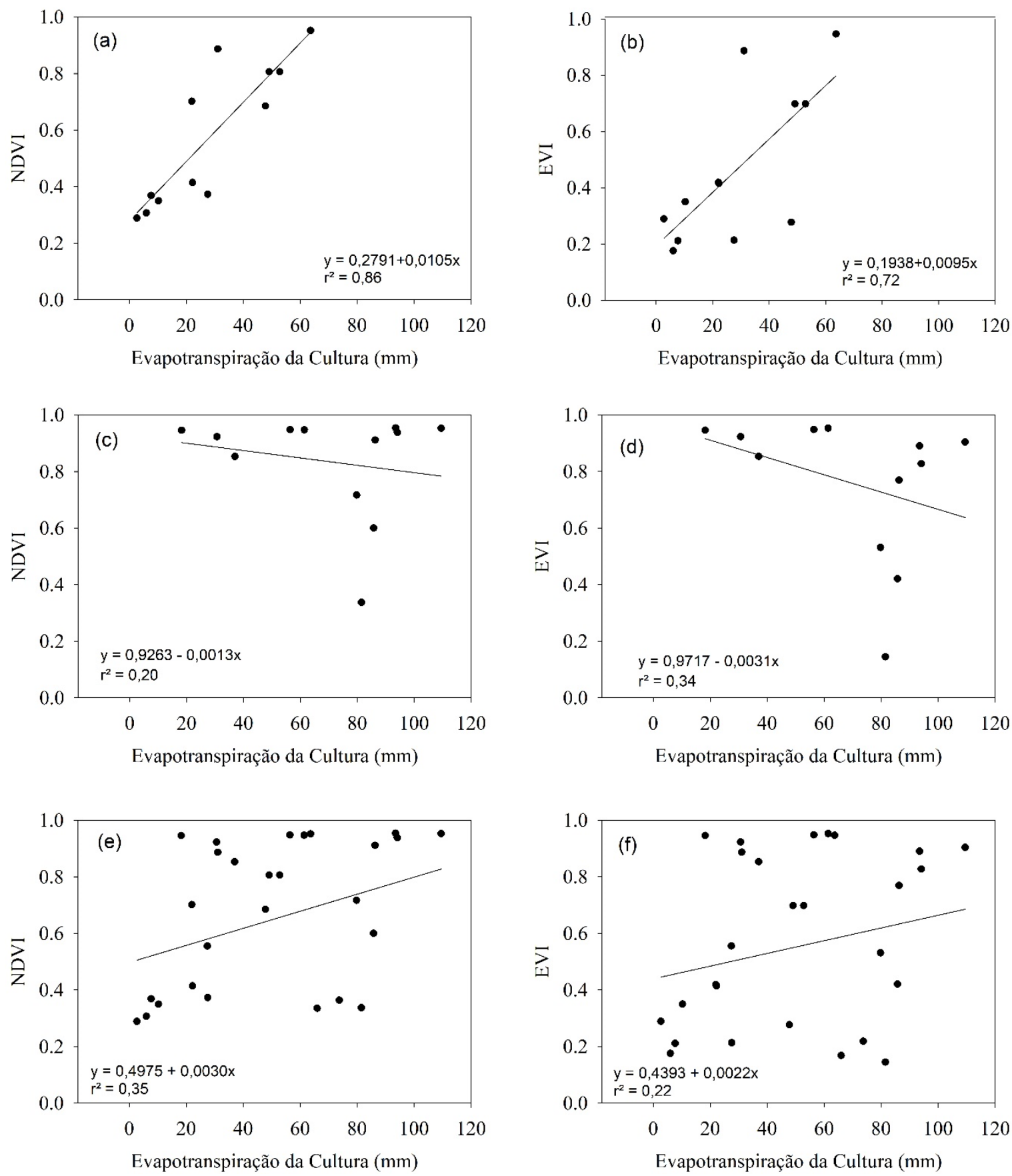

\section{Referências}

ALMEIDA, T. Respostas espectrais da soja sob diferentes condições hídricas e de preparo de solo. Dissertação (PPGSensoriamento Remoto UFRGS), 2008.

ALVARES, C. A.; STAPE, J. L.; SENTELHAS, P.C., de MORAES GONÇALVES, J.L.; SPAROVEK, G. Köppen's climate classification map for Brazil. Meteorologische Zeitschrift, [s.1.], v. 22, n. 6, p.711-728, 1 dez. 2013. Schweizerbart. http://dx.doi.org/10.1127/0941-2948/2013/0507
ARIAS, C. A.; OLIVEIRA, M.F de; PÍPOLO, A. E.; CARNEIRO, G.S.; UBIRAJAO RA, J.; KASTER, M. Desenvolvimento de cultivares de soja com tolerância à deficiência hídrica. Simpósio Sobre Tolerância à Deficiência Hídrica em Plantas, Santo Antonio de Goias, p.130-134, out. 2015.

BERGAMASCHI, H.; GUADAGNIN, M.R.; CARDOSO, L.S.; SILVA, M.I.G. Clima da Estação Experimental da UFRGS (e Região de Abrangência). Porto Alegre: UFRGS, 2003. 78p

BERLATO, M. A.; FONTANA, D. C. Variabilidade Interanual da precipitacao pluvial e rendimento da soja no Estado do Rio Grande do Sul. Revista de Agrometeorologia, Santa Maria, v. 7, n. 1, p.119-125, jul. 1999. 
CONAB. Bases de dados. Disponível em: http://www.conab.gov.br/. Acesso em 10 de set. 2018

COUTO, A. F.; CARVALHO Jr, O.A.; MARTINS, E.S.. Séries temporais MODIS aplicadas em sucessão de culturas de soja (Glycine max (L.) Merrill) e milho (Zea mays L.) em sistema de plantio direto. Revista Brasileira de Cartografia, v. 64, n. 3, p. 405-418, 2012

CUNHA, G. R., et al. Balanço Hídrico Climático. In: BERGAMASCHI, Homero et al. Agrometeorologia Aplicada a Irrigação. 2. ed. Porto Alegre: Editora da Universidade, 1992. Cap. 5. p. 63-85

CUNHA, G. R; BERGAMASCHI, H. Efeitos da disponibilidade hídrica sobre o rendimento das culturas. In: BERGAMASCHI, H. et al. Agrometeorologia aplicada à irrigação. Porto Alegre: UFRGS, 1992. Cap.6, p.85-97.

CUNHA, G. R; PASINATO, A. Análise agrometeorológica da safra de soja 2016/2017 em Passo Fundo, RS. Soja: resultados de pesquisa 2016/2017, p. 9, 2017

EMBRAPA. Exigências climáticas. In: EMBRAPA. Tecnologias de produção de soja - Paraná - 2003. Londrina: Embrapa Soja, 2002. Cap.1, p.28-30. (Sistemas de Produção; 2).

FARIAS, J. R. B.; ASSAD, E.D.; ALMEIDA, I.R.; EVANGELISTA, B.A.; LAZZAROTTO, C.; MEUMAIER, N.; NEPOMUCENO, A.L. Caracterização de risco de déficit hídrico nas regiões produtoras de soja no Brasil. Revista Brasileira de Agrometeorologia, Santa Maria, v. 9, n. 3, p. 415-421 2001

FIORI, D.; TONIOLO, G.; CUNHA, H.; FONTANA, D.C.; SALDANHA, D.S Relação entre NDVI e dados de precipitação em diferentes safras de soja no município de Cruz Alta - RS. Anais Xvii Simpósio Brasileiro de Sensoriamento Remoto - Sbsr, João Pessoa, v. 17, n. 1, p.6351-6357, abr. 2015. Disponível em: <http://www.dsr.inpe.br/sbsr2015/files/p1379. pdf>. Acesso em: 14 jun. 2019.

FREITAS, M. C. M. A cultura da soja no brasil: o crescimento da produção brasileira e o surgimento de uma nova fronteira agrícola. EnciclopÉdia Biosfera, Goiânia, v. 7, n. 12, p.1-12, 31 maio 2011. Disponível em: <http://conhecer.org.br/enciclop/2011a/agrarias/a\%20cultura\%20 da\%20soja.pdf>. Acesso em: 12 jun. 2019.

FONTANA, D. C.; SANTOS, L.N.; DALMAGO, G.A.; SCHIRMBECK, J. SCHIRMBECK, L.W. NDVI e alguns fatores de variabilidade. In: Anais do xix simpósio brasileiro de sensoriamento remoto, 2019, Santos. Anais eletrônicos... Campinas, GALOÁ, 2019. Disponível em: <https://proceedings.science/sbsr-2019/papers/ndvi-e-alguns-fatores-de-variabilidade> Acesso em: 14 jun. 2019.

HOOGENBOOM, G. Contribution of agrometeorology to the simulation of crop production and its application. Agricultural and Forest Meteorology, 103, 137-157, 2000.

HUETE, A.; DIDAN, K; MIURA, T.; RODRIGUEZ, E.P.; X GAO; FERREIRA, L.G., Overview of the radiometric and biophysical performance of the MODIS vegetation indices. Remote Sensing of Environment, [s.l.], v. 83, n. 1-2, p.195-213, nov. 2002. Elsevier BV. http://dx.doi.org/10.1016/ s0034-4257(02)00096-2. Disponível em: <https://doi.org/10.1016/S00344257(02)00096-2>. Acesso em: 17 set. 2019

JENSEN, J. R. Sensoriamento Remoto do Ambiente - Uma Perspectiva em Recursos Terrestres. Tradução da $2^{\mathrm{a}}$ edição J.C.N EPIPHANIO (org.). São José dos Campos: Parênteses Editora. 2011. Cap: 11 - Sensoriamento Remoto da Vegetação, p 357.

JOINER, J.; YOSHIDA, Y.; ANDERSON, M.; HOLMES, T.; HAIN, C.; REICHLE, R.; KOSTER, R.; MEDDLETON, E.; ZENG, F. Global relationships among traditional reflectance vegetation indices (NDVI and NDII), evapotranspiration (ET), and soil moisture variability on weekly timescales Remote Sensing. of Environment, Greenbelt, p.339-352, 24 out. 2018. Disponível em: <https://doi.org/10.1016/j.rse.2018.10.020>. Acesso em: 04 jun. 2019.
JUNGES, A. H.; FONTANA, D. C. Desenvolvimento das culturas de cereais de inverno no Rio Grande do Sul por meio de perfis temporais do índice de vegetação por diferença normalizada. Ciência Rural, Santa Maria , v. 39 , n. 5 , p. $1349-1355$

KATUL, G. G.; OREN, R.; MANZONI, S.; HIGGINS, C.; PARLANGE, M.B. Evapotranspiration: A process driving mass transport and energy exchange in the soil-plant-atmosphere-climate system. Reviews of Geophysics, [s.1.], v. 50, n. 3, p.1-25, 20 jul. 2012. American Geophysical Union (AGU). http://dx.doi.org/10.1029/2011rg000366.

MONTEIRO, J. E. B. A. (Org.). Agrometeorologia dos Cultivos: O fator meteorológico na produção agrícola. Brasilia: Inmet, 2009. 530 p. Disponível em: <http://www.inmet.gov.br/portal/css/content/home/publicacoes/agrometeorologia_dos_cultivos.pdf>. Acesso em: 14 jun. 2019.

MELO, R. W.; FONTANA, D.C.; BERLATO, M.A. Indicadores de produção de soja no Rio Grande do Sul comparados ao zoneamento agrícola. Pesquisa Agropecuária Brasileira, Brasilia, v. 36, n. 12, p.1167-1175, dez. 2004. Disponível em: <http://www.scielo.br/pdf/pab/v39n12/22857. pdf>. Acesso em: 14 jun. 2019.

MELO, R. W.; FONTANA, D.C.; BERLATO, M.A.; DUCATI, J.R. An agrometeorological-spectral model to estimate soybean yield, applied to southern Brazil. International Journal of Remote Sensing, [s.l.], v. 29, n. 14, p.4013-4028, 14 jun. 2008. Informa UK Limited. http://dx.doi. org/10.1080/01431160701881905.

PINTO, D. G.; FONTANA, D. C.; DALMAGO, G. A.; CUNHA, G.R.; FOCHESATO, E.; VICARI, M.B.; GOUVEA, J.A.; SANTI, A. Temporal dynamics of spectral reflectance and vegetation indices during canola crop cycle in southern Brazil. Ciência Rural, [s.1.], v. 47, n. 1, p.1-8, 2017. Disponível em: <http://dx.doi.org/10.1590/0103-8478cr20151403>. Acesso em: 17 set. 2019.

RIZZI, R.; RISSO, J.; EPIPHANIO, R.D.V.; RUDORFF, B.F.T.; FORMAGGIO, A.R.; SHIMABUKURO, Y.E.; FERNANDES, S.L. Estimativa da área de soja no Mato Grosso por meio de imagens MODIS, 2008. Anais XIV Simpósio Brasileiro de Sensoriamento Remoto, Natal, Brasil, 25-30 abril 2009, INPE, p. 387-394.

RISSO, J.; RIZZI, R; RUDORFF, B.F.T.; ADAMI, M.; SHIMABUKURO, Y.E.; FORMAGGIO, A.R.; EPIPHANIO, R.D.V. Índices de vegetação Modis aplicados na discriminação de áreas de soja. Pesquisa Agropecuária Brasileira, Brasilia, v. 47, n. 9, p.1317-1326, set. 2012. Disponível em: <https://www.researchgate.net/profile/Marcos_Adami/publication/267560026_Indices_de_vegetacao_Modis_aplicados_na_discriminacao_de_areas_de_soja/links/54626fb30cf2c0c6aec1b263.pdf>. Acesso em: 14 jun. 2019.

ROPPA KUSS, R. C., KONIG, O.; DUTRA, L.M.C.; BELLÉ, A.; ROGGIA, S. STURMER, G. Populações de plantas e estratégias de manejo de irrigacao na cultura da soja. Ciência Rural, vol. 38, no. 4, 2008, p. 1133+. Academic OneFile, https://link.galegroup.com/apps/doc/A193835793/ AONE? $u=$ ufrgs_br\&sid=AONE\&xid=c840fa72. Accessed 4 June 2019

ROUSE, J.W.; HAAS, R.H; SCHELL, J.A.; DEERING, D.W. Monitoring vegetation systems in the great plains with ERTS. 1973 In: Earth Resources Technology Satellite Symposium, 3., 1973, Washington. Proceedings... Washington: NASA. p.309-317.

SATVEG (Org.). SATVeg: Sistema de Análise Temporal da Vegetação. 2019. Disponível em: <https://www.satveg.cnptia.embrapa.br/satveg/ login.html>. Acesso em: 12 set. 2019

STRECK, E.V.; KÄMPF, N.; DALMOLIN, R. S. D ; KLAMT, E.; NASCIMENTO, P. C.; SCHNEIDER, P.; GIASSON, E.; PINTO L. F. S. Solos do Rio Grande do Sul. 2 ed. Porto Alegre: Emater/RS, 2008. 222 p.

THORNTHWAIT, C.W.; MATHER, J.R, Instructions and tables for computing potential evapotranspiration and the water balance. Publication in climatology, Centerton, New Jersey, 10(3): 181-311. 1955.

\section{REFERENCIAÇÃO}

SCHAPARINI, L. P.; FONTANA, D. C.; DALMAGO, G. A.;

RODIGHERI, G.; FERNANDES, J. M. C.; SANTI, A.; VARGAS; P. R. Analise comparativa entre índices de vegetação e sua relação com o balanço hídrico em soja. Agrometeoros, Passo Fundo, v.27, n.1, p.183190 , set 2019.

Declaração: os trabalhos estão sendo publicados nesse número de AGROMETEOROS (v.27, n.1, set 2019) conforme foram aceitos pelo XXI Congresso Brasileiro de Agrometeorologia, realizado de 12 a 16 de agosto de 2019, em Catalão, Goiás, sem revisão editorial adicional da revista. 


\title{
Comparative analysis between vegetation index and their relationship with soybean water balance
}

\author{
Laura Pigatto Schaparini ${ }^{1}$, Denise Cybis Fontana ${ }^{1,2(*)}$, Genei Antonio Dalmago ${ }^{3}$, Grazieli Rodigheri ${ }^{2}$, \\ José Mauricio Cunha Fernandes ${ }^{3}$, Anderson Santi ${ }^{3}$ and Paulo Roberto Vargas ${ }^{4}$ \\ ${ }^{1}$ Universidade Federal do Rio Grande do Sul - Faculdade de Agronomia. Avenida Bento Gonçalves 7712, Caixa Postal 15096, CEP 91501.970. Porto Alegre, \\ RS, Brazil. E-mails: laura_pigatto@yahoo.com.br e dfontana@ufrgs.br \\ ${ }^{2}$ Universidade Federal do Rio Grande do Sul, Programa de Pós-Graduação em Sensoriamento Remoto - PPGSR, Centro Estadual de Pesquisa em Senso- \\ riamento Remoto e Meteorologia - CEPSRM. Av. Bento Gonçalves, 9500 - Campus do Vale-Caixa Postal15044, CEP 91501-970 Porto Alegre, RS, Brazil. \\ E-mail: grazielirodigheri@gmail.com \\ ${ }^{3}$ Embrapa Trigo. Rodovia BR 285, km 294, Caixa Postal 3081, CEP 99050-970 Passo Fundo, RS, Brazil. E-mails: genei.dalmago@embrapa.br, mauricio. \\ fernandes@embrapa.br e anderson.santi@embrapa.br \\ ${ }^{4}$ Fazenda Capão Grande. Carazinho, RS, Brazil. E-mail: paulovargas@annex.com.br \\ ${ }^{(*)}$ Corresponding author.
}

\section{ARTICLE INFO}

Article history:

Received 17 September 2019

Accepted 4 March 2020

\section{Index terms:}

soybean

water conditions

NDVI

EVI

evapotranspiration

\section{ABSTRACT}

Soybean is a legume of annual cycle and of great economic importance in Rio Grande do Sul. For this crop, stresses caused by water deficiency are responsible for instability in yield and grain production. One way to monitor water deficiency is through remote sensing, which has been widely used in agriculture, most often as vegetation index (IVs). The objective of this study was to evaluate the temporal pattern of NDVI and EVI index for three soybean harvests, with different water conditions, in order to allow the evaluation of the relationship between IVs and water conditions for the crop. The area of the experiment On Farm was located in the municipality of Carazinho, RS, Brazil. In the field was installed a meteorological station to measure environmental variables. Daily water balance was calculated for each crop taking into account crop evapotranspiration (ETc). The IVs were taken from the Satveg platform. In addition to the graphical analysis, correlation analysis was performed between soybean IVs and ETc. Both NDVI and EVI were strongly related to plant vegetative development, varying similarly throughout the soybean cycle. Both index had the same pattern of profile oscillations in periods of water deficit. NDVI and EVI can be used to evaluate soybean crop water condition

(C) 2020 SBAgro. All rights reserved.

\section{CITATION \\ SCHAPARINI, L. P.; FONTANA, D. C.; DALMAGO, G. A.; \\ RODIGHERI, G.; FERNANDES, J. M. C.; SANTI, A.; VARGAS; P. R. Analise comparativa entre índices de vegetação e sua relação com o balanço hídrico em soja. Agrometeoros, Passo Fundo, v.27, n.1, p.183- 190, set 2019.}

